\title{
TINTES NATURALES VEGETALES EN EL PARAJE EL DESMONTE, RESERVA CULTURAL-NATURAL CERRO COLORADO, CÓRDOBA (ARGENTINA)
}

\author{
M. FERNANDA PAVÁN ${ }^{1}$, VIOLETA FURLAN ${ }^{2}$, MAURICIO RENNY ${ }^{1-3}$, IGNACIO MONTERROSO ${ }^{1}$ \& LILIANA ARGÜELLO ${ }^{1}$
}

\begin{abstract}
Summary: Paván, M. F., V. Furlan, M. Renny, I. Monterroso \& L. Argüello. 2017. Natural plant dyes in El Desmonte, Cerro Colorado Natural and Cultural Reserve, Córdoba (Argentina). Bonplandia 26(2): 103-113.
\end{abstract}

\begin{abstract}
Non-timber forest products have high intrinsic value as well as ecological, cultural and scientific value. Dye plants are among these resources whose knowledge and use in Cordoba is concentrated in the rural communities of small cattle producers in the Northwest and North of the Province. In this work the objective is to acknowledge the historical and current use of dye plants and the associated practices that allow to rescue and to revalue environmental knowledge of the north of Cordoba, particularly in the Paraje El Desmonte, Cerro Colorado Natural-Cultural Reserve. We inquired about the local knowledge to obtain and use the natural dyes of plants in the studied area and compared the dye species from native forests with those used in EI Desmonte. Data were obtained through ethnobiological tools. There were 24 potential dye species in the area, with records of historical use in other sites in northwestern Cordoba. In the study area, seven species are currently used. It was evidenced that forests are still an important supplier of these resources and that the use of dye plants combined with dyeing techniques is a current practice which was passed on for generations.
\end{abstract}

Key words: Environmental knowledge, non-timber forest products (NTFPs), rural inhabitants.

\begin{abstract}
Resumen: Paván, M. F., V. Furlan, M. Renny, I. Monterroso \& L. Argüello. 2017. Tintes naturales vegetales en el paraje El Desmonte, Reserva Cultural-Natural Cerro Colorado, Córdoba (Argentina). Bonplandia 26(2): 103-113.
\end{abstract}

Los productos forestales no maderables tienen valor intrínseco, alto valor ecológico, cultural y científico. Las plantas tintóreas se encuentran entre estos recursos cuyo conocimiento y uso en Córdoba se concentra en las comunidades rurales de pequeños productores ganaderos del noroeste y norte provincial. En este trabajo el objetivo es dar a conocer el uso histórico y actual de especies tintóreas y las prácticas de reproducción social asociadas que permitan rescatar y revalorizar saberes ambientales del norte cordobés, en particular, en el Paraje El Desmonte, Reserva Cultural-Natural Cerro Colorado. Se indagó acerca de los saberes locales para la obtención y uso de los tintes naturales del área de estudio y se comparó las especies tintóreas presentes en bosque nativo y las utilizadas en el paraje. Los datos fueron obtenidos mediante la implementación de herramientas etnobiológicas. Se registraron 24 especies tintóreas potenciales en la zona, con registros de uso histórico en otros sitios del noroeste cordobés. En el área de estudio se utilizan actualmente 7 de ellas. Se evidenció que los bosques siguen siendo

\footnotetext{
${ }^{1}$ Cátedra de Problemática Ambiental. Facultad de Ciencias Exactas, Físicas y Naturales, Universidad Nacional de Córdoba. E-mail: laferpavan2014@gmail.com

${ }^{2}$ Instituto de Biología Subtropical, Universidad Nacional de Misiones-Consejo Nacional de Investigaciones Científicas y Técnicas, Puerto Iguazú.

${ }^{3}$ Instituto Multidisciplinario de Biología Vegetal, IMBIV, UNC-CONICET, Argentina.
} 
un importante proveedor de estos productos y el uso de plantas tintóreas aunado a las técnicas de obtención de los tintes es una práctica vigente y aprendida de manera intergeneracional.

Palabras clave: Productores rurales, productos forestales no maderables (PFNM), saberes ambientales.

\section{Introducción}

En América latina y el Caribe, desde la época prehispánica, los productos forestales no maderables (PFNM) han proporcionado materiales básicos para el desarrollo de las poblaciones rurales y contribuido a la estabilidad de los agro-ecosistemas locales (Bustos \& Bonino, 2005). Entre ellos podemos destacar especies que cubren necesidades de diferentes tipos, tales como especies alimenticias, medicinales, de importancia cultural, con potencial económico. Estas funcionalidades pueden ocurrir por separado o en simultáneo dependiendo de las especies. $\mathrm{Su}$ buen aprovechamiento puede contribuir tanto a mejorar los ingresos de los pobladores rurales como a la conservación de los bienes naturales (Cordeiro-Beduschi et al., 2006).

Entre los PFNM se encuentran las plantas tintóreas, especies vegetales que se emplean para la producción de tintes naturales con los que se tiñen hilados, tejidos, cueros e inclusive la piel humana. Plantas tintóreas se encuentran en todo el mundo y, en cuanto a su cantidad y calidad, América es un continente particularmente rico (Marzocca, 1959). Si bien existen tintes naturales de origen vegetal, animal o mineral, cabe aclarar que en este trabajo cuando se habla de "tintes naturales" se hace referencia a los obtenidos de plantas o sea los derivados de origen vegetal.

La utilización de tintes naturales se conoce desde tiempos prehistóricos, para embellecer y adornar diferentes artículos de uso corriente (Marzocca, 1959), para pintar el cuerpo y los alimentos, teñir la vestimenta, entre otras (Suárez \& Arenas, 2012). La utilización de tintes también se ha asociado con momentos de importancia en la vida de una persona o de un grupo. Por ejemplo, Arenas (2004) menciona Bixa orellana L. "achiote" (Bixaceae) y Genipa americana L. "genipapo" (Rubiaceae), utilizadas por su valor ceremonial para pinturas corporales en grupos chaqueños. Para extraer los tintes naturales vegetales se utilizan diferentes técnicas como la maceración, el machacado, la disolución en agua o diversos solventes, separación por decantación, desecación, entre otras (Marzocca, 2005).

A partir del siglo XX, particularmente en las ciudades, los tintes naturales cedieron terreno frente a los sintéticos, debido a que estos últimos producen colores más llamativos y brillantes, a la diversificación de las gamas de colores posibles (Palacio, 2007) y al hecho de que su fijación es más sencilla (Lambaré et al., 2011). Los colorantes sintéticos de la industria moderna fueron acogidos por el público, por considerarlos de mejor calidad y menor costo que los escasos tintes naturales (Cedano et al., 2001). De este modo, se fue perdiendo el uso de algunos tintes naturales, junto con sus técnicas o procedimientos de tinción (Flores \& Ling, 1990; Gutiérrez et al., 2006). El siglo XXI, sin embargo, comienza con una motivación por recuperar los tintes vegetales locales (Palacio et al., 2007; Keller, 2010; Suárez \& Arenas, 2012), asociada a las crisis económicas y a la revalorización de las artesanías locales, sumado a la toma de conciencia sobre la toxicidad y contaminación que producen los colorantes sintéticos. Además, en la Argentina, los productores de tejidos tradicionales aprecian la posibilidad de aumentar el valor agregado a sus productos a partir del uso de tintes naturales (Trillo et al., 2007).

En la provincia de Córdoba, el conocimiento y uso de plantas tintóreas se concentra en las comunidades rurales de pequeños productores ganaderos del noroeste y norte provincial (Trillo et al., 2007). Los pequeños productores conservan tecnologías ancestrales y también innovaciones para la obtención y producción de tintes, que aportan a la diversificación de sus ingresos. Sin embargo, las comunidades tradicionales que poseen el conocimiento sobre plantas y técnicas apropiadas para la tinción, por múltiples causas, pierden rápidamente la información en el contexto 
de su integración creciente al mercado global (Trillo et al., 2007). A partir de esto se planteó el objetivo de conocer la historia y el uso actual de especies tintóreas y sus prácticas de reproducción social en el Paraje El Desmonte, Córdoba, permitiendo rescatar y revalorizar los saberes ambientales del norte cordobés. A su vez, se relevó la comunidad boscosa de las inmediaciones del paraje para identificar las especies tintóreas presentes y poder mensurar la potencialidad de obtener otras especies tintóreas del bosque.

\section{Materiales y Métodos}

\section{Área de estudio}

El área de estudio se circunscribe a un predio privado correspondiente a una familia del Paraje El Desmonte, ubicado a $160 \mathrm{~km}$ al norte de la ciudad de Córdoba, entre los $63^{\circ} 92^{\prime}$ y $63^{\circ} 94^{\prime}$ de longitud y los $30^{\circ} 06^{\prime}$ y $30^{\circ} 07^{\prime}$ de latitud, en la falda oriental de la porción intermedia de la Sierra del Norte, 5 $\mathrm{km}$ al norte de la localidad de Cerro Colorado, Dep. Río Seco, Córdoba, Argentina. El predio familiar forma parte de la Reserva Cultural-Natural Cerro Colorado (Decreto Nro. 2821/92) constituyendo una de las áreas núcleo de la misma, aunque una parte del área de estudio forma parte también de la zona de amortiguamiento de la Reserva (Fig. 1). En el área relevada convergen el Dominio climático semi-desértico de las planicies del noroeste y el Dominio semi-seco, con tendencia al semihúmedo (Capitanelli, 1979). Sin embargo, la presencia del Cordón montañoso Cerro Colorado actúa como barrera orográfica, haciendo que las precipitaciones sean más abundantes $(600-850 \mathrm{~mm})$ respecto de las descriptas para ambos Dominios (Herrero, 2000). El predio familiar ocupa la porción media y final de la subcuenca del arroyo Pozanjón del Norte y de la subcuenca La Quebrada, que desembocan al sur en el río Los Tártagos, siendo la cuenca de este último una de las más extendidas de la Sierra Norte, que lleva agua todo el año (Herrero, 2000). Luti et al. (1979), al igual que Cabrera (1971) describen la vegetación del sector como perteneciente a la Provincia Fitogeográfica Chaqueña, Distrito
Chaqueño Serrano Oriental, y distinguen la singularidad de la Sierra del Norte de entre el resto de las Sierras de Córdoba por la escasa presencia de Schinopsis marginata Engl. "orco quebracho" (Anacardiaceae) y la dominancia de Myrcianthes cisplatensis (Cambess.) O. Berg "mato" (Myrtaceae). El Paraje El Desmonte es uno de los lugares de Cerro Colorado con registros más antiguos de población, previo a la existencia de la localidad de Cerro Colorado y de la Reserva (Argüello et al., 2004). Las formas principales de subsistencia de los pobladores se distribuyen entre la cría a pequeña y mediana escala de animales, principalmente de ganado vacuno y ovino, el empleo en pequeños comercios, la fabricación de productos artesanales y manufacturas. Asimismo, el empleo público ocupa un porcentaje importante de los pobladores locales, a través de trabajos en el ámbito de la Reserva, de la comuna y de la escuela, además de las jubilaciones y asistencia pública a la vejez (Arias Toledo, 2006).

\section{Obtención de datos}

El relevamiento de los datos se realizó entre los meses de octubre de 2010 a marzo de 2011, con una periodicidad de viajes cada 15 días durante los meses de octubre a diciembre, quedándose en el lugar por 5 días en cada viaje. Desde enero a marzo la primera autora se estableció en el lugar conviviendo con los pobladores durante esos 3 meses con el objetivo de lograr un trabajo de campo en profundidad y con fuerte vinculación en la localidad. Los datos fueron obtenidos mediante herramientas etnobiológicas usuales, como entrevistas semiestructuradas a informantes clave. En un comienzo se entrevistó a cada familia del paraje (5 familias) y luego se decidió trabajar con una de ellas en profundidad por su gran conocimiento en la temática y ser reconocidos como referentes locales. A través de las entrevistas se recuperaron conocimientos relativos a las especies vegetales empleadas para teñir, sus nombres locales, las partes empleadas en el proceso de teñido y los colores obtenidos. Se indagó, además, acerca del proceso de obtención de los tintes naturales y del teñido en general, el tipo de materiales que se somete a coloración y el 


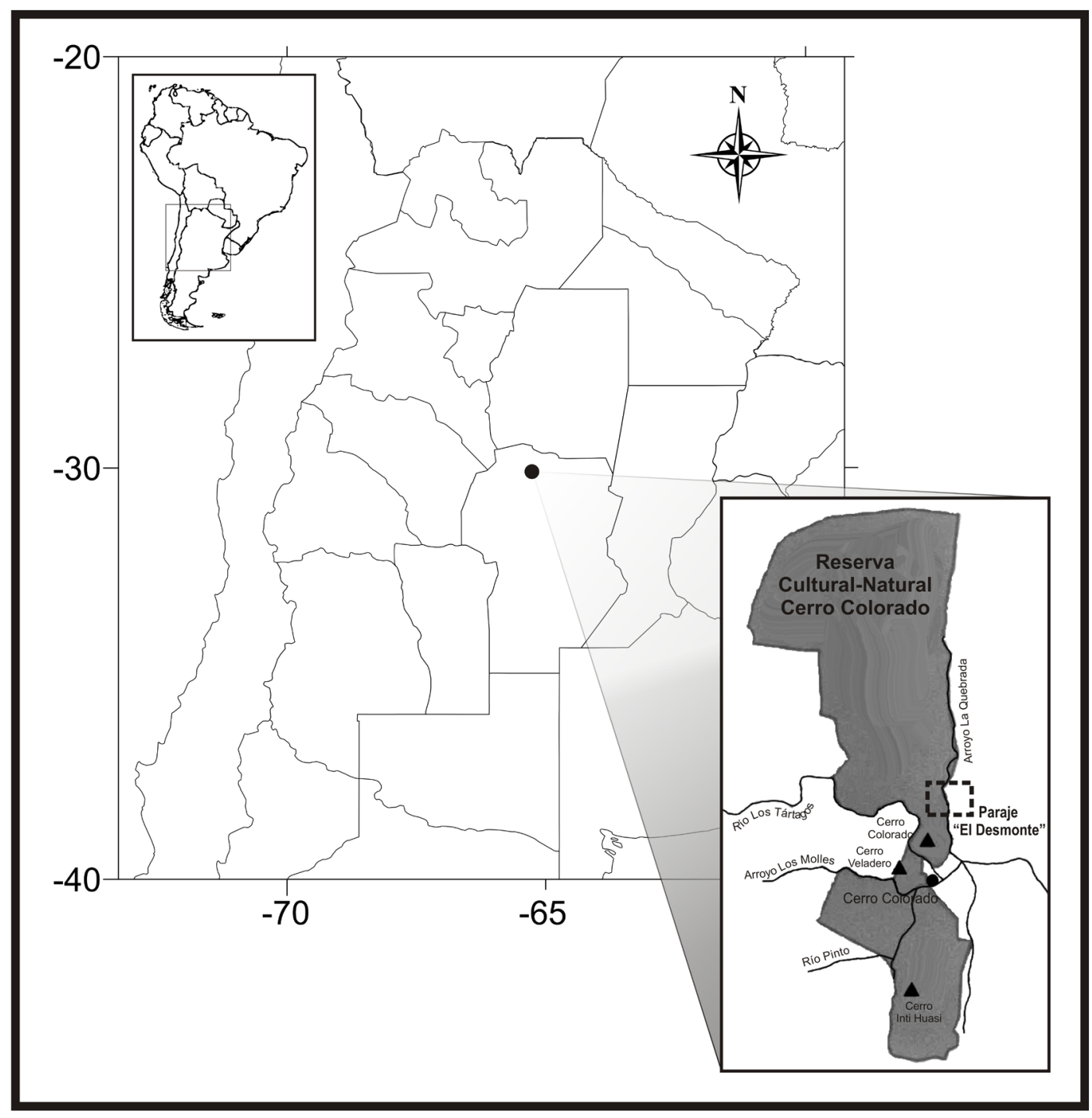

Fig. 1. Ubicación geográfica de la Reserva Cultural-Natural Cerro Colorado dentro del territorio argentino. Ampliación del área que muestra la Reserva en toda su extensión y la ubicación del Paraje El Desmonte.

Fig. 1. Geographic location of the Cerro Colorado Natural-Cultural Reserve within Argentine territory. The expanded figure shows the Reserve in all its extension and the location of the Paraje El Desmonte.

uso o no de mordientes. También se realizaron caminatas con las personas entrevistadas en los bosques utilizados para obtener las especies tintóreas con el fin de identificarlas botánicamente. Además, de las caminatas con los entrevistados, en diferentes instancias se pudo presenciar y participar del proceso de obtención de los tintes, el teñido de la lana y la confección de prendas. Así la primera autora pudo aprender de primera mano las técnicas utilizadas tanto para el teñido de la lana como para el manejo del telar.

Por otra parte, se utilizó información cuantitativa obtenida partir de relevamientos florísticos, con el objetivo de poder dilucidar las potencialidades tintóreas de las plantas del bosque. Para este punto, se detectaron a partir de fotos áreas las diferentes comunidades 
vegetales presentes en el lugar, en las cuales se realizaron transectas tipo Canfield, ubicadas al azar, perpendiculares al sentido de la pendiente; su tamaño y número se determinó a través de curvas de rango-abundancia (MuellerDombois \& Ellenberg, 1974; Matteucci \& Colma, 1982).

Luego se hizo una búsqueda bibliográfica para dar cuenta de cuáles especies estaban registradas como recursos tintóreos, a partir de la compilación realizada por Trillo \& Demaio (2007). A partir de esto, se realizaron listas de especies tintóreas del área de estudio, comparándose la lista relevada mediante las transectas y las determinadas como tintóreas de acuerdo a los resultados de Trillo \& Demaio (2007) (Tabla 2), y las utilizadas por la familia de productores (Tabla 1). Las especies tintóreas se colectaron dentro del predio familiar en los lugares de recogida habituales de los pobladores. Su determinación botánica se realizó en las Instalaciones del Centro de Ecología y Recursos Naturales Renovables (CERNAR) y en la Cátedra de Problemática Ambiental de la Facultad de Ciencias Exactas, Físicas y Naturales de la Universidad Nacional de Córdoba. Primero las especies fueron herborizadas a campo y luego de su determinación se depositaron ejemplares testigo de las especies que se mantienen en el herbario de trabajo de la cátedra. Los nombres científicos de cada una fueron corroborados con la versión on-line del Catálogo de las Plantas Vasculares del Cono Sur (Zuloaga et al., 2017).

\section{Resultados}

La actividad textil y tintórea en el área de estudio

Una de las actividades más importantes del grupo familiar entrevistado es la textil. La familia mantiene un proceso tradicional en el manejo de la lana y es la más antigua en el sector que trabaja con tintes naturales.

La actividad textil y tintórea llega al predio familiar de la mano de la jefa de hogar, productora oriunda del Puesto de Los Bulacio, ubicado en el camino que une las localidades de Cerro Colorado y Rayo Cortado (a unos 6 $\mathrm{km}$ del Paraje El Desmonte). La productora (informante clave) es una referente local que ha enseñado a productores que trabajan con tintes en la zona. A su vez, ha difundido su conocimiento en el sector a través de talleres de tintes, por lo cual su técnica es ampliamente utilizada.

A partir de la información brindada en las entrevistas y observación participante se

Tabla 1. Especies vegetales tintóreas utilizadas en el área de estudio por los productores. *Equivale a "médula" en la terminología botánica.

Table 1. Dye plants used in the study area by the producers. *Equals "pith" in botanical terminology.

\begin{tabular}{|c|c|c|c|c|}
\hline Familias & Especies & $\begin{array}{l}\text { Nombres } \\
\text { Vulgares }\end{array}$ & $\begin{array}{l}\text { Partes } \\
\text { Utilizadas }\end{array}$ & Colores \\
\hline Asteraceae & Baccharis coridifolia DC. & Romerillo & Ramas & $\begin{array}{l}\text { Amarillo (verde } \\
\text { con herrumbre) }\end{array}$ \\
\hline Fabaceae & Prosopis nigra (Griseb.) Hieron. & Algarrobo negro & Corazón* & Marrón oscuro \\
\hline Fabaceae & Acacia caven (Molina) Molina & Espinillo & Corazón* & Marrón claro \\
\hline Myrtaceae & $\begin{array}{l}\text { Myrcianthes cisplatensis } \\
\text { (Cambess.) O. Berg }\end{array}$ & Mato & $\begin{array}{l}\text { Corteza y } \\
\text { Corazón* }\end{array}$ & $\begin{array}{l}\text { Rosado (gris con } \\
\text { herrumbre) }\end{array}$ \\
\hline Solanaceae & Cestrum parqui L'Hér. & Duraznillo negro & Fruto & Verde \\
\hline Rhamnaceae & Condalia buxifolia Reissek & $\begin{array}{l}\text { Piquillín de la } \\
\text { Sierra }\end{array}$ & Corazón* & Marrón rojizo \\
\hline Rutaceae & $\begin{array}{c}\text { Zanthoxylum coco Gillies ex } \\
\text { Hook. f. \& Arn. }\end{array}$ & Coco & Corteza & Verde \\
\hline
\end{tabular}


Tabla 2. Especies vegetales tintóreas presentes en el área de estudio según datos bibliográficos. *En Trillo \& Demaio, 2007 Ziziphus mistol Griseb.

Table 2. Dye plants present in the study area according to bibliographic data. *In Trillo \& Demaio, 2007 Ziziphus mistol Griseb.

\begin{tabular}{|c|c|c|c|c|}
\hline Familias & Especies & $\begin{array}{l}\text { Nombres } \\
\text { Vulgares }\end{array}$ & $\begin{array}{l}\text { Partes } \\
\text { Utilizadas }\end{array}$ & Colores \\
\hline Anacardiaceae & Lithraea molleoides (Vell.) Engl. & $\begin{array}{l}\text { Molle de } \\
\text { beber }\end{array}$ & Corteza y raíz & Marrón \\
\hline Anacardiaceae & $\begin{array}{c}\text { Schinus fasciculatus (Griseb.) I. M. } \\
\text { Johnst. }\end{array}$ & Moradillo & Corteza & No mencionado \\
\hline Apocynaceae & $\begin{array}{c}\text { Aspidosperma quebracho-blanco } \\
\text { Schltdl. }\end{array}$ & $\begin{array}{l}\text { Quebracho } \\
\text { blanco }\end{array}$ & Corteza & $\begin{array}{l}\text { Rosado; } \\
\text { amarillo }\end{array}$ \\
\hline Asteraceae & Achyrocline satureioides (Lam.) DC. & Vira-vira & Toda la planta & No mencionado \\
\hline Asteraceae & Bacharis crispa Spreng. & Carqueja & Toda la planta & Amarillo \\
\hline Asteraceae & Bacharis coridifolia DC. & Romerillo & Toda la planta & Amarillo \\
\hline Asteraceae & Bidens pilosa L. & Amor seco & Toda la planta & Amarillo \\
\hline Asteraceae & Tagetes minuta L. & Suico & Toda la planta & $\begin{array}{l}\text { Amarillo-verde; } \\
\text { marrón-verde }\end{array}$ \\
\hline Asteraceae & Zinnia peruviana (L.) L. & $\begin{array}{l}\text { Flor de } \\
\text { papel }\end{array}$ & $\begin{array}{l}\text { No } \\
\text { mencionada }\end{array}$ & Verde \\
\hline Celtidaceae & $\begin{array}{c}\text { Celtis ehrenbergiana (Klotzsch) } \\
\text { Liebm. }\end{array}$ & Tala & Tallo & Marrón \\
\hline Cactaceae & Cereus forbesii Otto ex C. F. Först. & Ucle & Fruto & Rosado \\
\hline Fabaceae & Acacia caven (Molina) Molina & Espinillo & Fruto & Marrón claro \\
\hline Fabaceae & $\begin{array}{c}\text { Geoffrea decorticans (Gillies ex Hook. } \\
\text { \& Arn.) Burkart }\end{array}$ & Chañar & Corteza & No mencionado \\
\hline Fabaceae & Prosopis alba Griseb. & $\begin{array}{l}\text { Algarrobo } \\
\text { blanco }\end{array}$ & Resina y tallo & Marrón \\
\hline Fabaceae & $\begin{array}{c}\text { Prosopis chilensis (Molina) Stuntz } \\
\text { emend. Burkart }\end{array}$ & $\begin{array}{l}\text { Algarrobo } \\
\text { blanco }\end{array}$ & Resina y tallo & Marrón \\
\hline Myrtaceae & $\begin{array}{c}\text { Myrcianthes cisplatensis (Cambess.) } \\
\text { O. Berg }\end{array}$ & Mato & $\begin{array}{l}\text { Corteza y } \\
\text { duramen }\end{array}$ & Rosado \\
\hline Phytolaccaeae & Rivina humilis $\mathrm{L}$. & $\begin{array}{l}\text { Sangre de } \\
\quad \text { toro }\end{array}$ & Fruto & Rojo \\
\hline Polygonaceae & Ruprechtia apetala Wedd. & $\begin{array}{l}\text { Manzano } \\
\text { del campo, } \\
\text { Higuerrilla }\end{array}$ & Corteza y raíz & $\begin{array}{c}\text { Marrón rojizo y } \\
\text { bayo }\end{array}$ \\
\hline Rhamnaceae & Colletia spinossisima J. F. Gmel. & $\begin{array}{l}\text { Barba de } \\
\text { tigre }\end{array}$ & Toda la planta & $\begin{array}{l}\text { Amarillo } \\
\text { verdoso }\end{array}$ \\
\hline Rhamnaceae & Condalia buxifolia Reissek & $\begin{array}{l}\text { Piquillín de } \\
\text { la sierra }\end{array}$ & Raíz & Marrón rojizo \\
\hline Rutaceae & $\begin{array}{c}\text { Zanthoxylum coco Gillies ex Hook. f. } \\
\text { \& Arn. }\end{array}$ & Coco & Tallo & Amarillo y verde \\
\hline Solanaceae & Cestrum parqui (Lam.) L'Hér. & $\begin{array}{l}\text { Duraznillo } \\
\text { negro }\end{array}$ & Fruto & Verde \\
\hline Verbenaceae & $\begin{array}{c}\text { Aloysia gratissima (Gillies \& Hook. ex } \\
\text { Hook.) Tronc. var. gratissima }\end{array}$ & $\begin{array}{l}\text { Palo } \\
\text { amarillo }\end{array}$ & Tallo & Amarillo \\
\hline Zygophyllaceae & $\begin{array}{c}\text { Sarcomphalus mistol (Griseb.) } \\
\text { Hauenschild* }\end{array}$ & Mistol & Corteza y raíz & Marrón rojizo \\
\hline
\end{tabular}


destaca que el proceso de producción de lanas y tintes, y la elaboración de mantas, es una actividad manual artesanal intergeneracional donde participa toda la familia. La recolección de las plantas tintóreas y la obtención de los tintes es realizada por el jefe del hogar en ocasiones acompañado por uno de sus hijos, y el hilado y la obtención de las prendas al igual que los diseños son generados por la jefa del hogar. Según los productores, los lugares de recolección de las plantas son siempre los mismos. Entre los factores que destacan para la selección de estos sitios se mencionan la importancia de estar en las cercanías a la vivienda y la abundancia de las especies.

\section{Producción de tejidos a escala local}

El procedimiento utilizado para la producción textil consta de cuatro pasos: 1) obtención de la lana, 2) obtención de tintes, $3)$ teñido de la lana y 4) confección de la prenda. La obtención de los tintes se inicia con la tarea de recolección de las plantas necesarias para teñir, que se seleccionan en función del color que se desea obtener. La lana se obtiene de esquilar el ganado ovino propio de cada familia. Los tintes a partir de árboles y arbustos nativos de la zona, siendo utilizados con preferencia ramas caídas o árboles debilitados, que se cortan y se extrae el "corazón" (en términos locales) o médula, que luego se trituran y se colocan en agua dentro de un recipiente de uso exclusivo. Una vez obtenido el tinte, se coloca la lana en el recipiente y se lleva a hervor, se retira del fuego y se deja estacionar hasta el día siguiente. Luego, el producto se enjuaga con agua y se coloca al sol para secar. Una

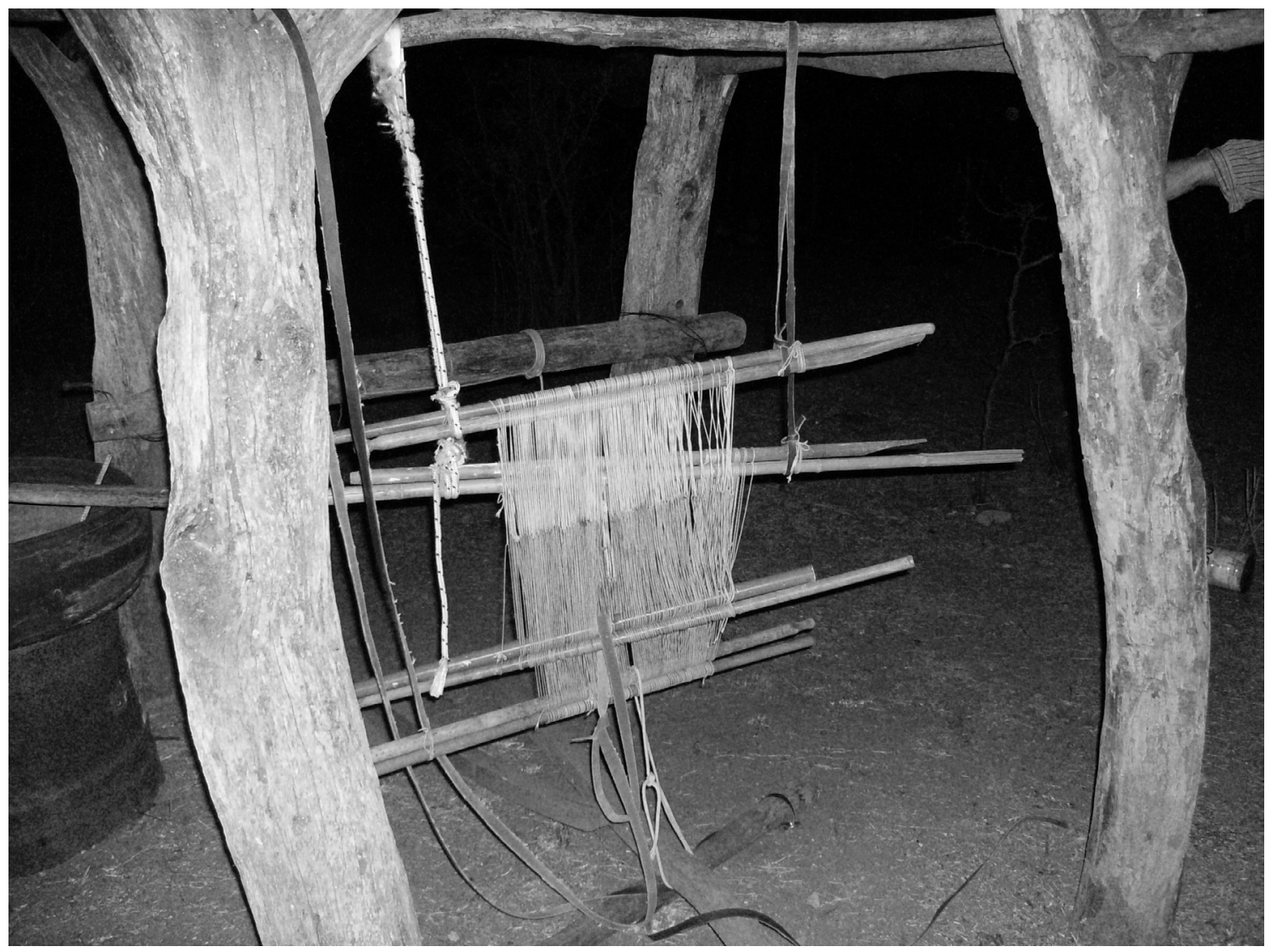

Fig. 2. Telar de pala (telar criollo) de la familia, ubicado en el patio de la vivienda.

Fig. 2. Shovel loom (creole loom) of the family, located in the backyard of the house. 


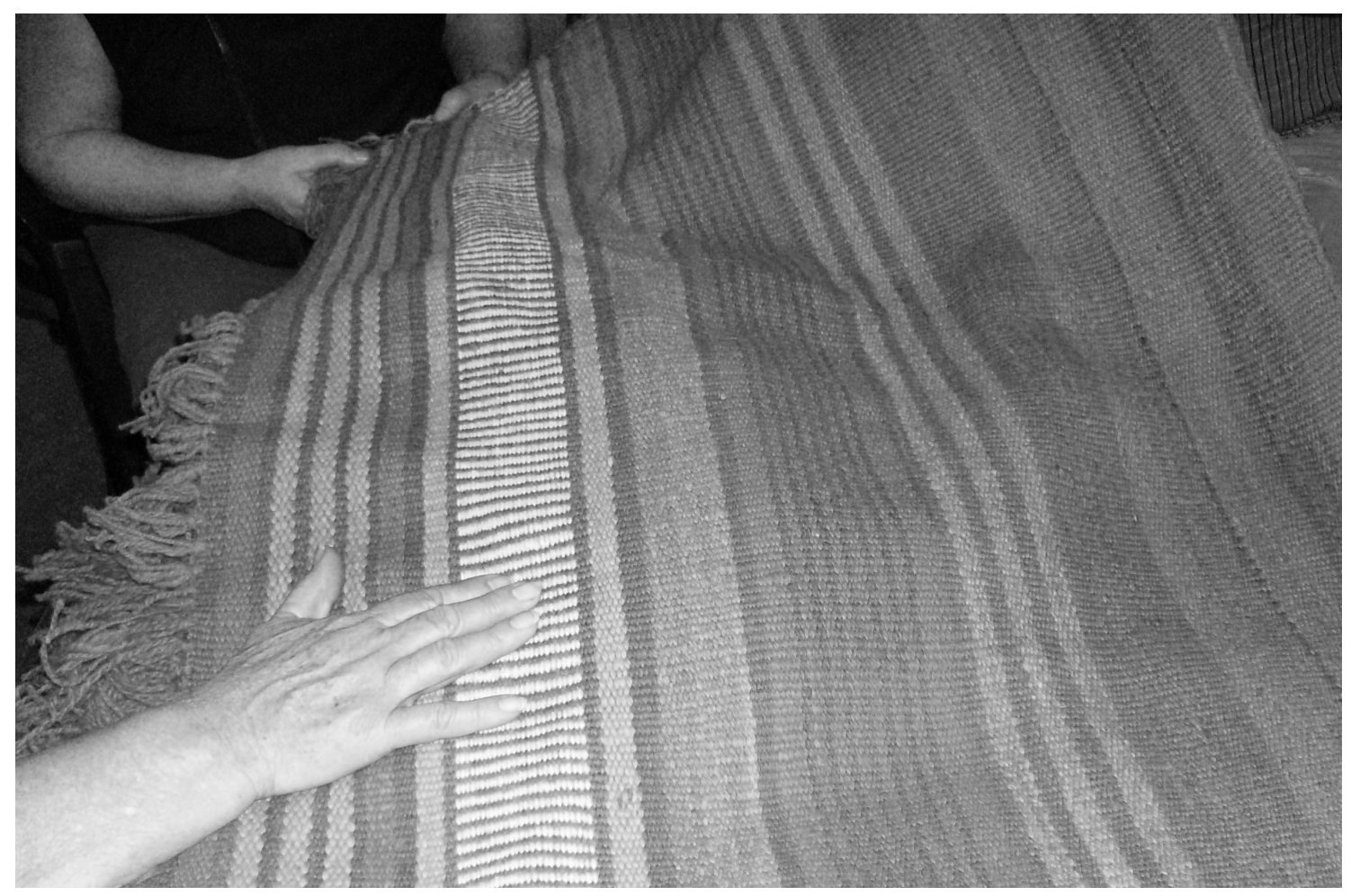

Fig. 3. Técnicas de diseño: rayas y peines. Diseños realizados por la productora local.

Fig. 3. Design techniques: stripes and comb patterns. Designs performed by a local producer.

vez teñida la lana se procede a realizar las prendas por medio del telar criollo. Las familias del paraje lo llaman "telar de pala", por la presencia de un palo o pala de madera que es un instrumento que forma parte del telar, con el cual se golpea la urdimbre, y se lo utiliza para ajustar los hilos y hacer un tejido parejo y producir telas compactas. Tanto la utilización del telar como las técnicas usadas para los diseños, rayas y peines pertenecen a cada familia. Los diseños y telar que se muestran en las figuras 2 y 3 provienen de la tradición familiar de la principal entrevistada y referente local. Además del trabajo en telar, que genera ponchos, mantas, alforjas y fajas de uso tradicional en el campo, la actividad turística que se desarrolla en Cerro Colorado, y los esfuerzos de entidades públicas y privadas de generar una oferta de productos locales, han llevado a las familias locales a diversificar la oferta de productos generando carteras, ruanas, caminos de mesa, entre otros. Existen también innovaciones en el tratamiento de la lana, en la generación de nuevos diseños de las prendas y en la incorporación de pinturas rupestres a los tejidos manuales, como también el uso combinado de tintes naturales y sintéticos.

\section{Especies tintóreas utilizadas y potenciales}

De las especies tintóreas presentes en el Paraje El Desmonte la familia propietaria utiliza siete para producir los tintes, de los cuales se obtienen seis colores diferentes (Tabla 1). En el predio familiar consiguen diversos colores: verde, amarillo, anaranjado, marrón, gris, rosado y gamas de los mismos. Las especies utilizadas son todas nativas y se distribuyen en seis familias botánicas. Muchas veces se usan los tintes básicos para producir otros colores, o variantes del mismo color, para lo cual se utilizan mordientes como cenizas o herrumbre (óxido de hierro). Este último, es utilizado con dos especies, Myrcianthes 
cisplatensis, "mato" (Myrtaceae) y Baccharis coridifolia DC. "romerillo" (Asteraceae), el primero vira de rosado a gris y el segundo de verde a amarillo.

El relevamiento de las comunidades vegetales y la recopilación bibliográfica posibilitaron detectar 24 especies tintóreas en el área de estudio distribuidas en 14 familias botánicas con predominio de las familias Asteraceae $(26,09 \%)$ y Fabaceae $(17,39 \%)$ (Tabla 2$)$. La comparación entre las especies potencialmente tintóreas y las utilizadas permitió conocer que los productores entrevistados utilizan un $29 \%$ de las mismas.

\section{Discusión}

En el norte argentino, en general, la tarea textil y tintórea es realizada por mujeres (Palacio, 2007; Lambaré et al., 2011; Suárez \& Arenas, 2012) y son ellas las que juegan un papel crucial en la adquisición y transmisión de conocimientos (Lambaré et al., 2011). En El Desmonte, en coincidencia, es la jefa del hogar quien ha traído las actividades al paraje $\mathrm{y}$ es también quien ha impartido talleres de tintes en la zona. Esto ha generado el interés en el paraje y zonas aledañas y fomentado el uso de las plantas tintóreas con el objetivo por parte de la artesana de que se conserven las técnicas y se transmitan las enseñanzas. Esto concuerda con lo expuesto por Lambaré et al. (2011) en cuanto a la perspectiva de los artesanos de fomentar los talleres para que las técnicas no queden en el olvido.

Como se mencionó en resultados, el turismo en el sector así como el contacto con otros artesanos ha alentado a diversificar la oferta de bienes, lo cual ha implicado generar nuevos productos, diseños y mezclas de anilinas y tintes naturales. Esto último también se ha observado en productos generados en otras comunidades del norte de la Argentina (Lambaré et al., 2011; Suárez \& Arenas, 2012). Esta diversificación de productos ha influido de manera positiva en el ingreso monetario familiar.

Al igual que en otras comunidades de la provincia fitogeográfica Chaqueña, los productos artesanales son realizados casi exclusivamente con lana de oveja (Palacio, 2007) y la parte del vegetal tradicionalmente empleada en la obtención de los tintes naturales es el tallo triturado (Fabbio et al., 2009), aunque en El Desmonte se utiliza la médula en vez de la corteza. Además, la técnica de impregnación de los colores en lana (Martínez, 2012) y el tipo de telar, conocido con el nombre de "telar criollo", es citado para otras comunidades de la provincia (Palacios, 2007).

Las especies tintóreas utilizadas por la principal familia entrevistada se encuentran, en su mayoría, dentro de la lista de especies tintóreas citadas para el Distrito Chaqueño Serrano (Trillo \& Demaio, 2007), destacándose los bosques como fisonomía predominante en la obtención de los tintes naturales. Entre las especies utilizadas se encuentran el "algarrobo negro", Prosopis nigra (Griseb.) Hieron., especie no registrada por Trillo \& Demaio (2007) pero si señalada por otros autores (Carrizo et al., 2006; Palacio, 2007) para el Distrito Chaqueño, de la cual se obtienen tintes de la gama de los marrones (Carrizo et al., 2006; Palacio, 2007).

La recolección de las especies en Cerro Colorado se realiza alrededor de la vivienda, tal como lo describe Palacio (2007) para otra comunidad del Distrito Chaqueño. Sin embargo, esta caracterización tiene una excepción, el "romerillo", Baccharis coridifolia, que crece entre los 500 y $2500 \mathrm{~m}$ s.n.m. (Sérsic et al., 2006), por lo cual tiene que ser buscado en su lugar de distribución.

Según la parte de la planta usada, así como del mordiente utilizado en la tinción, es posible obtener diferentes colores y gamas. Los mordientes utilizados por la familia se encuentran dentro de los más comunes utilizados en el pasado (Lambaré et al., 2011), quedando en evidencia como se resguardan algunos conocimientos ancestrales.

Con respecto a las potenciales especies tintóreas, la familia botánica más representada es Asteraceae. Cabe mencionar lo citado por diferentes autores (Marzocca, 1959; Del Vitto \& Petenatti, 2009) sobre la importancia del uso etnobotánico que siempre se ha hecho de diferentes especies de Asteraceae como plantas tintóreas. 


\section{Conclusiones}

A partir de los resultados obtenidos se evidencia que los bosques peri-domiciliarios siguen siendo un importante proveedor de materia prima para la producción de tintes. $\mathrm{Su}$ mantenimiento en el Paraje El Desmonte es fundamental, siendo utilizadas las especies vegetales dominantes del bosque. El uso de plantas tintóreas y las técnicas de obtención de los tintes es una práctica antigua que sigue vigente en el área y que ha tenido un momento de expansión, según lo explicitado por los propios pobladores, asociado a la industria turística del lugar. La transmisión de los conocimientos relacionados con la forma de obtención de los tintes se da tanto de manera intergeneracional como entre pares. Esto último, representado a partir de los talleres brindados por la familia productora a otras familias y tejedoras que quieren comenzar con la actividad.

Las plantas tintóreas y los tintes obtenidos de las mismas ostentan gran importancia para los seres humanos formando parte de la historia e identidad de diferentes culturas, por lo cual su conservación es una de las formas de mantener vivas las costumbres y la memoria de los pueblos involucrados así como la biodiversidad asociada. Esto es relevante para Cerro Colorado, donde la valoración del entorno natural y cultural está dada tanto por los pobladores locales como por la creación de la Reserva.

Para futuros estudios se plantea trabajar con aquellas tejedoras que fueron formadas por la familia productora, para investigar si las técnicas aprehendidas y los colores utilizados se mantienen, o se ha ampliado la gama de estrategias de tinción. A su vez, sería interesante trabajar en medidas para el manejo sustentable de las plantas tintóreas del lugar. Así como para realizar un bosque de tintóreas en las inmediaciones de los hogares.

\section{Agradecimientos}

A la familia de productores, en especial a Américo y Nora, por habernos permitido recorrer cada espacio de su tierra y de su historia. A la Cátedra de Problemática Ambiental por sus aportes y espacio físico. A la Dra. Marta Ojeda por la lectura cuidadosa y crítica del artículo.

Este manuscrito está dedicado a la memoria de Jessica Zarate...Siempre que pensamos en las plantas se nos viene a la mente la imagen de tus manos en la tierra...

\section{Bibliografía}

ARENAS, P. 2004. Los vegetales en el arte del tatuaje de los indígenas del Gran Chaco. En CIPOLLETTI, M. S. (ed.), Los mundos de arriba y los mundos de abajo. Individuo y sociedad en las tierras bajas, en los Andes y más allá, pp. 249-274. ABYA YALA, Quito, Ecuador.

ARGÜELLO, L., G. SCHWINDT, C. BARTÓ, C. \& M. GONELLA. 2004. Impacto de las actividades humanas en los sistemas boscosos de la Reserva Natural-Cultural Cerro Colorado y su región de influencia. Córdoba. Sinergia.

ARIAS TOLEDO, B. 2006 Aspectos cuantitativos, cualitativos y simbólicos de la medicina tradicional de los pobladores criollos de Cerro Colorado (Córdoba, Argentina). Invest. Antropol. Cogn., PINACO 4: 105-115.

BUSTOS, J. \& E. BONINO 2005. Cosecha silvestre de peperina (Minthosthachys mollis) en Córdoba, Argentina: implicancias socioeconómicas. Rev. Ibeoram Econ. Ecol. 2: 45-55.

CABRERA, A. L. 1971. Fitogeografía de la República Argentina. Bol. Soc. Argent. Bot. 14: 1-42.

CAPITANELLI, R. 1979. Geomorfología. En VÁZQUEZ, J. B., R. A. MIATELLO \& M. E. ROQUÉ (eds.), Geografía Física de la Provincia de Córdoba, pp. 213-296. Ed. BOLDT, Córdoba, Argentina.

CARrizO, E., M. O. PALACIO, L. D. ROIC, A. A.VILLAVERDE, M. L. SORIA \& M. A. TORRES. 2006. Utilización de especies de Prosopis L. por pobladores rurales en Santiago del Estero, Argentina. Ponencia en Jornadas de la Facultad de Ciencias Forestales-UNSE. http://fcf.unse.edu.ar/eventos/2jornadas-forestales/pdfs/Utilizacion $\% 20 \mathrm{de} \% 20$ Prosopis.pdf

CEDANO, M., L. VILLASEÑOR \& L. GUZMÁNDÁVALOS. 2001. Some Aphyllophorales tested for organic dyes. Mycologist 15: 81-85.

CORDEIRO-BEDUSCHI, L. E., J. PALMA, R. PEDRAZA \& A. ROMERO. 2006. Manejo comunitario de productos forestales no madereros: Los desafíos de comunidades indígenas en Chile. III Encuentro de la "Associação Nacional de PósGraduação e Pesquisa em Ambiente e Sociedade" 
(ANPPAS). Brasilia, Brasil, 23 al 26 de Mayo de 2006. pp. 1-16.

DEL VITTO, L. A. \& E. M. PETENATTI. 2009. Asteráceas de importancia económica y ambiental. Primera parte. Sinopsis morfológica y taxonómica, Importancia ecológica y Plantas de interés industrial. Multequina 18: $87-115$

FABBIO, F. A., N. I. HILGERT \& D. A. LAMBARÉ. 2009. Los tintes naturales de Los Toldos y alrededores. $1^{\circ}$ Ed. CYTED-RISAPRET, San Salvador de Jujuy, $90 \mathrm{pp}$.

FLORES, L. \& F. LING. 1990. Artesanía en Talamanca: El Sémko y los Colorantes Naturales. CATIE. Turrialba, CR. 7 pp.

GUTIÉRREZ, N., S. DÍAZ, J. YEOMANS \& C. HERNÁNDEZ. 2006. Manual de tintes de origen natural para papel con fibra de pinzote de banano. Tierra Tropical 2: A1-A30.

HERRERO, S. 2000. Procesos sedimentarias Holocenos en la cuenca del río Los Tártagos (Sierra Norte, Provincia de Córdoba): Implicancias paleoclimáticas y geomorfológicas. Tesis Doctoral, Facultad de Ciencias Exactas Físicas y Naturales, Universidad Nacional de Córdoba, Argentina, 200 pp.

KELLER, H. A. 2010. Plantas colorantes utilizadas por los Guaraníes de Misiones, Argentina. Bonplandia 19: 11-25.

LAMBARÉ, D. A., N. I. HILGERT \& R. S. RAMOS. 2011. Dyeing plants and knowledge transfer in the Yungas communities of Northwest Argentina. Econ. Bot. 65: 315 .

LUTI, R., M. A. BERTRÁN DE SOLÍS, F. M. GALERA, N. MÜELLER DE FERREYRA, M. BERZAL, M. NORES, M. A. HERRERA \& J. C. BARRERA. 1979. Vegetación. En VÁZQUEZ, J. B., R. A. MIATELLO \& M. E. ROQUÉ (eds.). Geografía Física de la Provincia de Córdoba, pp. 297-368. Ed. BOLDT. Argentina.

MARTÍNEZ, G. J. 2012. Recolección, disponibilidad y uso de plantas en la actividad artesanal de comunidades tobas (qom) del Chaco Central, Argentina. En Arenas, P. (ed.) Etnobotánica en zonas áridas y semiáridas del cono sur de Sudamérica, pp.195-223. Edición CEFYBO-CONICET, Buenos Aires.

MARZOCCA, A. 1959. Historia de plantas tintóreas y curtientes. Colección Agropecuaria del INTA. Buenos
Aires, Argentina, 234 pp.

MARZOCCA, A. 2005. Plantas exóticas colorantes o tintóreas cultivadas en la Argentina. Academia Nacional de Agronomía y Veterinaria. Buenos Aires, $130 \mathrm{pp}$.

MATTEUCCI, S. D. \& A. COLMA. 1982. Metodología para el estudio de la Vegetación. Secretaría General de la O.E.A. Programa Regional de Desarrollo Científico y Tecnológico. Monografía No 22. Washington, D. C., 163 pp.

MUELLER-DOMBOIS, D. \& H. ELLENBERG. 1974. Aims and methods of vegetation ecology. John Wiley $\&$ Sons, New York, 547 pp.

PALACIO, M. 2007. El uso de los recursos vegetales con propiedades tintóreas en la industria artesanal familiar en dos departamentos de la provincia de Santiago del Estero, República Argentina. Tesis de Magister. Universidad Nacional de Santiago del Estero, Argentina, $87 \mathrm{pp}$.

PALACIO, M., E. CARRIZO \& L. ROIC. 2007. Relevamiento de plantas tintóreas utilizadas en localidades del Dpto. Atamisqui (Santiago del Estero, Argentina). Kurtziana 33: 73-78

SÉRSIC, A., A. COCUCCI, S. BENÍTEZ-VIEYRA, A. COSACOV, L. DÍAZ, E. GLINOS, N. GROSSO, C. LAZARTE, M. MEDINA, M. MORÉ, M. MOYANO, J. NATTERO, V. PAIARO, C. TRUJILLO \& P. WIEMER. 2006. Flores del centro de Argentina. Una guía ilustrada para conocer 141 especies típicas. Academia Nacional de Ciencias. Córdoba, Argentina, $354 \mathrm{pp}$.

SUÁREZ, M. E. \& P. ARENAS. 2012. Plantas y hongos tintóreos de los wichís del Gran Chaco. Bol. Soc. Argent. Bot. 47: 275-283.

TRILLO, C. \& P. DEMAIO. 2007. Tintes naturales. Guía para el reconocimiento y uso de plantas tintóreas del Centro de Argentina. Ediciones Sezo, Córdoba, Argentina, 79 pp.

TRILLO, C., P. DEMAIO, S. COLANTONIO \& L. GALETTO. 2007. Conocimiento actual de plantas tintóreas por los pobladores del valle de Guasapampa, provincia de Córdoba. Kurtziana 33: 65-71.

ZULOAGA, F. O., O. MORRONE \& M. J. BELGRANO (eds.). 2017. Catálogo de las Plantas Vasculares del Cono Sur. Disponible en http://www.darwin.edu.ar/ Proyectos/FloraArgentina/fa.htm [Acceso: 2017].

Original recibido el 5 de septiembre de 2017; aceptado el 6 de octubre de 2017. 
